\title{
The hOCT1 and $A B C B 1$ polymorphisms do not influence the pharmacodynamics of nilotinib in chronic myeloid leukemia
}

\author{
Sara Galimberti ${ }^{1}$, Cristina Bucelli ${ }^{2}$, Elena Arrigoni ${ }^{3}$, Claudia Baratè ${ }^{1}$, Susanna \\ Grassi $^{1,4}$, Federica Ricci ${ }^{1}$, Francesca Guerrini ${ }^{1}$, Elena Ciabatti ${ }^{1}$, Carmen Fava ${ }^{5}$, \\ Antonio D'Avolio ${ }^{6}$, Giulia Fontanelli' ${ }^{1}$ Giovanna Rege Cambrin', Alessandro \\ Isidori ${ }^{8}$, Federica Loscocco ${ }^{8}$, Giovanni Caocci ${ }^{9}$, Marianna Greco ${ }^{9}$, Monica Bocchia ${ }^{10}$, \\ Lara Aprile ${ }^{10}$, Antonella Gozzini ${ }^{11}$, Barbara Scappini ${ }^{11}$, Daniele Cattaneo ${ }^{2}$, Anna \\ Rita Scortechini ${ }^{12}$, Giorgio La Nasa9 ${ }^{2}$ Alberto Bosi ${ }^{11}$, Pietro Leoni ${ }^{12}$, Romano \\ Danesi $^{3}$, Giuseppe Saglio ${ }^{5}$, Giuseppe Visani ${ }^{8}$, Agostino Cortelezzi ${ }^{2}$, Mario Petrini ${ }^{1}$, \\ Alessandra Iurlo ${ }^{2}$ and Antonello Di Paolo ${ }^{3}$

\footnotetext{
${ }^{1}$ Department of Clinical and Experimental Medicine, Section of Hematology, University of Pisa, Pisa, Italy

${ }^{2}$ Oncohematology Division, IRCCS Ca' Granda, Maggiore Policlinico Hospital Foundation, University of Milan, Milano, Italy

${ }^{3}$ Department of Clinical and Experimental Medicine, Section of Pharmacology, University of Pisa, Pisa, Italy

${ }^{4}$ GeNOMEC, University of Siena, Siena, Italy

${ }^{5}$ Hematology Division, Ospedale Mauriziano, Torino, Italy

${ }^{6}$ Laboratory of Clinical Pharmacology and Pharmacogenetics, Department of Medical Sciences, University of Torino, Torino, Italy

${ }^{7}$ Department of Clinical and Biological Sciences, University of Torino, AOU San Luigi Gonzaga, Torino, Italy

${ }^{8}$ Hematology and Stem Cell Transplant Center, San Salvatore Hospital, Pesaro, Italy

${ }^{9}$ Department of Medical Sciences, University of Cagliari, Cagliari, Italy

${ }^{10}$ Division of Hematology, Ospedale Le Scotte, University of Siena, Siena, Italy

${ }^{11}$ Division of Hematology, AOU Careggi, University of Florence, Firenze, Italy

${ }^{12}$ Division of Hematology, Marche Polytechnic University, Ancona, Italy
}

Correspondence to: Sara Galimberti, email: sara.galimberti@med.unipi.it

Keywords: nilotinib, $A B C$ transporters, $A B C B 1$, hOCT1, early molecular response

Received: June 24, $2017 \quad$ Accepted: August 29, $2017 \quad$ Published: September 30, 2017

Copyright: Galimberti et al. This is an open-access article distributed under the terms of the Creative Commons Attribution License 3.0 (CC BY 3.0), which permits unrestricted use, distribution, and reproduction in any medium, provided the original author and source are credited.

\section{ABSTRACT}

First-line nilotinib in chronic myeloid leukemia is more effective than imatinib to achieve early and deep molecular responses, despite poor tolerability or failure observed in one-third of patients. The toxicity and efficacy of tyrosine kinase inhibitors might depend on the activity of transmembrane transporters. However, the impact of transporters genes polymorphisms in nilotinib setting is still debated. We investigated the possible correlation between single nucleotide polymorphisms of hOCT1 (rs683369 [c.480C>G]) and ABCB1 (rs1128503 [c.1236C>T], rs2032582 [c.2677G >T/A], rs1045642 [C.3435C $>$ T] ) and nilotinib efficacy and toxicity in a cohort of 78 patients affected by chronic myeloid leukemia in the context of current clinical practice. The early molecular response was achieved by $81 \%$ of patients while $64 \%$ of them attained deep molecular response (median time, 26 months). The 36-month event-free survival was $86 \%$, whereas $58 \%$ of patients experienced toxicities. Interestingly, hOCT1 and $A B C B 1$ polymorphisms alone or in combination did not influence event-free survival or the adverse events rate. Therefore, in contrast to data obtained in patients treated with imatinib, hOCT1 and ABCB1 polymorphisms 


\section{do not impact on nilotinib efficacy or toxicity. This could be relevant in the choice of the first-line therapy: patients with polymorphisms that negatively condition imatinib efficacy might thus receive nilotinib as first-line therapy.}

\section{INTRODUCTION}

After the introduction in the clinical practice of the tyrosine kinase inhibitors (TKIs), the outcome of patients affected by chronic myeloid leukemia (CML) has really improved, with $96 \%$ of them alive and free of progression at 3 years [1].

Nilotinib was initially approved as a secondgeneration TKI for the treatment of patients resistant to imatinib, and since 2007 also as a first-line option [2]. It is structurally similar to imatinib, binding to the inactive conformation of the protein encoded by the $B C R-A B L 1$ fusion gene, but it is also characterized by a higher efficacy than imatinib [3]. The prospective, randomized phase-3 trial ENESTnd comparing nilotinib $300 \mathrm{mg}$ or $400 \mathrm{mg}$ twice/day vs imatinib $400 \mathrm{mg}$ daily clearly demonstrated the superiority of nilotinib in terms of complete cytogenetic response (CCyR) (by 12 months, $80 \%$ in the nilotinib vs $65 \%$ in the imatinib arm) and of major molecular response (MR3) (by 5 years, 77\% for nilotinib vs $60.4 \%$ for imatinib, $p<0.0001$ ). Interestingly, progressions occurred in 5 out of the 563 patients in the nilotinib arms $(0.88 \%)$ in comparison to 12 out of 283 cases receiving imatinib (4.2\%) [4].

Overall, the choice of nilotinib as first-line treatment significantly increases the probability of reaching the desired surrogate end-points at the correct time-points, but also the probability of achieving a deep molecular response (DMR, by 5 years, $54 \%$ with nilotinib vs $31 \%$ for imatinib), which represents the basis for a further possible drug discontinuation. Nevertheless, about $40 \%$ of patients discontinue nilotinib due to suboptimal response or toxicity [4].

Different mechanisms have been recognized on the basis of TKI resistance, from the $A B L 1$ mutations [5] to the activation of several alternative pro-proliferative pathways (such as Wnt, PI3K, Aurora Kinase, STAT3) [6$8]$ or the inhibition of the correct immunological control [9]. In addition, the transmembrane transporters have been also indicated as responsible for the resistance to TKIs, with a relevant role recognized to the ATP binding cassette $(\mathrm{ABC})$ efflux pumps and to the human organic cation transport member 1 (hOCT1) influx protein [10]. Indeed, the pharmacokinetics and pharmacodynamics of imatinib seem to be influenced by $A B C B 1$ [11-13], hOCT1 [14-16], and $A B C G 2$ polymorphisms [17]. Moreover, an in vitro study conducted on resistant K562 cells showed that $A B C G 2$ over-expression was associated with a reduced efficacy of imatinib, nilotinib, dasatinib, and bosutinib [18]. On the contrary, other studies demonstrated that dasatinib is a substrate of $A B C B 1$ [19], while the binding affinity of this transmembrane transporter seems to be lower for nilotinib [20, 21], bosutinib [22], and ponatinib [21]. Moreover, nilotinib appeared as one of the most potent modulators of $\mathrm{ABCB} 1$ and $\mathrm{ABCG} 2$ in respect of imatinib, dasatinib, erlotinib, lapatinib, and sunitinib in a murine model of chemoresistance [23].

Finally, a recent meta-analysis clearly demonstrated that the highest values of minimum plasma concentrations of imatinib were significantly correlated with the complete cytogenetic response, but not with the achievement of the complete molecular response [13]. About polymorphisms, authors concluded that the c.421A $A B C G 2$ variant allele was significantly associated with higher rate of MR3, whereas $A B C B 1$ variant alleles did not, with the exception of c.2677T/A alleles [13]. However, the complexity of the methods used for data analyses and the different noncomparable models have frequently led to conflicting results $[24,25]$.

With these premises, we decided to address the influence of $A B C B 1$ and $h O C T 1$ polymorphisms on the response rate and toxicity of first-line nilotinib in the context of a multicenter "real-life" series of $78 \mathrm{CML}$ cases. Of note, patients were enrolled in a prospective and retrospective way in order to perform the analyses in a powered study.

\section{RESULTS}

\section{Nilotinib efficacy: hematological, cytogenetic, molecular responses, and EFS}

Seventy-eight CML patients, all in chronic phase, median age 47 years (range, 18-79) were enrolled in the study. Forty-six (59\%) were males, and 32 (41\%) were females. According to the Sokal, Hasford, and European Treatment and Outcome Study (EUTOS) scores, nearly half of the patients were classified into the low-risk groups. The median follow-up time in our study was 43 months; $2 / 3$ of the enrolled cases were followed for 36 months, $74 \%$ for 24 months, $80 \%$ for 18 months. All patients had a minimum follow-up of 12 months. No significant differences in clinical characteristics have been observed between the prospective and retrospective cohorts (Table 1). The response rates have been detailed in Table 2.

\section{Hematological response}

Seventy-six out of the 78 patients enrolled into the study (97.4\%) achieved a complete hematological response (CHR) by 3 months of treatment; the remaining two subjects reached a complete response by the sixth month of therapy. Overall, the CHR rate in our series was $100 \%$. 
Table 1: Clinical characteristics of the 78 enrolled patients

\begin{tabular}{|c|c|c|c|c|}
\hline Clinical feature & Overall series n. (\%) & $\begin{array}{c}\text { Prospective cohort } \\
\text { n. }(\%)\end{array}$ & $\begin{array}{c}\text { Retrospective cohort } \\
\text { n. (\%) }\end{array}$ & $\begin{array}{c}\text { Statistical } \\
\text { significance }\end{array}$ \\
\hline Patients & 78 & 29 & 49 & \\
\hline Age $(\text { years) })^{* *}$ & $47(18-79)$ & $49(20-68)$ & $46(18-79)$ & $\mathrm{p}=0.460$ \\
\hline Sex & & & & $\mathrm{p}=0.351$ \\
\hline M & $46(59 \%)$ & $15(52 \%)$ & $31(63 \%)$ & \\
\hline $\mathrm{F}$ & $32(41 \%)$ & $14(48 \%)$ & $18(27 \%)$ & \\
\hline Sokal risk score & & & & $\mathrm{p}=0.542$ \\
\hline Low & $34(44 \%)$ & $11(38 \%)$ & $23(47 \%)$ & \\
\hline Intermediate & $29(37 \%)$ & $12(42 \%)$ & $17(34 \%)$ & \\
\hline High & $15(19 \%)$ & $6(20 \%)$ & $9(19 \%)$ & \\
\hline Eutos risk score & & & & $\mathrm{p}=0.301$ \\
\hline Low & $40(51 \%)$ & $11(38 \%)$ & $29(59 \%)$ & \\
\hline High & $27(35 \%)$ & $11(38 \%)$ & $16(33 \%)$ & \\
\hline N/A & $11(14 \%)$ & $7(24 \%)$ & $4(8 \%)$ & \\
\hline Hasford risk score & & & & $\mathrm{p}=0.054$ \\
\hline Low & $40(51 \%)$ & $11(38 \%)$ & $29(59 \%)$ & \\
\hline Intermediate & $26(34 \%)$ & $14(48 \%)$ & $12(25 \%)$ & \\
\hline High & $12(15 \%)$ & $4(14 \%)$ & $8(16 \%)$ & \\
\hline
\end{tabular}

Note: ", Students' $t$ test; **, values are expressed as median (min-max range)

\section{Cytogenetic response}

Sixty-nine patients $(88.5 \%)$ achieved a complete cytogenetic response (CCyR) by 12 months, with 66 cases $(84.6 \%)$ reaching a CCyR already by the sixth month [the time point identified as "optimal" by the European Leukemia Net (ELN) guidelines] [26]. The median time to achieve a CCyR was 3.2 months (range, 1.8-14.4); interestingly, by the $18^{\text {th }}$ month of treatment, the Philadelphia chromosome disappeared in 77 out of 78 patients. With a median follow-up of 43 months (range 4-106), no cases of loss of CCyR have been reported. The CCyR achievement was not significantly conditioned by sex, age, or Sokal and EUTOS score; nevertheless, the high Hasford score was associated with a lower probability of reaching the CCyR by the $12^{\text {th }}$ month with respect to the other groups $(11 \%$ vs $43 \%$; $=0.01)$. No significant differences in the quality of cytogenetic response were observed between the prospective and retrospective cohort.

\section{Molecular responses}

overall, the early molecular response (EMR) was achieved by 63 patients (81\%); then, 68 subjects $(87 \%)$ achieved an MR3 during the treatment. Of note, 60 patients reached an MR3 by 12 months, which identifies the optimal response. Furthermore, patients who experienced EMR more frequently achieved an MR3 by the $12^{\text {th }}$ month $(83.6 \%$ vs $38.5 \%$; $=0.002)$. The median time to achieve an MR3 was 6.1 months (range, 2.6 - 83); this interval was 7 months longer for cases without EMR. Seven patients (9\%) lost the MR3 during treatment, with a median time of 31.2 months. As reported in Table 2, the EMR rate was higher in the prospective than in the retrospective cohort $(\mathrm{p}=0.012)$.

The $64.1 \%$ of patients receiving nilotinib achieved a DMR ( $\geq$ MR4), with a median time to DMR attainment of 26 months: in particular, $24.3 \%, 28.2 \%$ and $11.5 \%$ of patients achieved an MR4, MR4.5 and MR5, respectively. As above reported for the MR3, also the DMR was more frequently achieved by subjects in EMR (71.8\% vs $35.8 \% ; \mathrm{p}=0.04)$. As reported in Table 2 , the DMR rate was higher in the retrospective than in the prospective cohort $(\mathrm{p}=0.002)$. The molecular response rates were not significantly conditioned by age, sex or CML risk scores.

\section{EFS}

All patients were still alive in July 2016. Thus, only the event-free survival (EFS) was considered for the statistical analysis. The 24-months EFS for the whole series was $89 \%$, while the EFS at 36 and 48 months was $86 \%$ and $81 \%$, respectively. Age $>60$ years, sex, and CML 
Table 2: Hematological, cytogenetic, and molecular responses at different time-points

\begin{tabular}{|c|c|c|c|c|}
\hline Responses & Overall Series n. (\%) & $\begin{array}{c}\text { Prospective cohort } \\
\text { n. }(\%)\end{array}$ & $\begin{array}{c}\text { Retrospective cohort } \\
\text { n. }(\%)\end{array}$ & $\begin{array}{c}\text { Statistical } \\
\text { significance }\end{array}$ \\
\hline \multicolumn{5}{|c|}{ CHR by 3 months } \\
\hline Yes & $76(97 \%)$ & $29(100 \%)$ & $47(96 \%)$ & $\mathrm{p}=0.392$ \\
\hline No & $2(3 \%)$ & 0 & $2(4 \%)$ & \\
\hline \multicolumn{5}{|c|}{ CCyR by 6 months } \\
\hline Yes & $66(85 \%)$ & $25(86 \%)$ & $41(84 \%)$ & $\mathrm{p}=0.376$ \\
\hline No & $12(15 \%)$ & $4(14 \%)$ & $8(16 \%)$ & \\
\hline \multicolumn{5}{|c|}{ CCyR by 12 months } \\
\hline Yes & $69(88.5)$ & $25(86 \%)$ & $44(90 \%)$ & $\mathrm{p}=0.593$ \\
\hline No & $9(11.5)$ & $4(14 \%)$ & $5(10 \%)$ & \\
\hline \multicolumn{5}{|l|}{ EMR } \\
\hline Yes & $63(81 \%)$ & $28(96 \%)$ & $35(71 \%)$ & $\mathrm{p}=\mathbf{0 . 0 1 2}$ \\
\hline No & $15(19 \%)$ & $1(4 \%)$ & $14(29 \%)$ & \\
\hline \multicolumn{5}{|c|}{ MR3 by 12 months } \\
\hline Yes & $60(77 \%)$ & $22(76 \%)$ & $38(78 \%)$ & $\mathrm{p}=0.537$ \\
\hline No & $18(23 \%)$ & $7(24 \%)$ & $11(22 \%)$ & \\
\hline \multicolumn{5}{|c|}{ MR3 (at any time) } \\
\hline Yes & $68(88 \%)$ & $23(79 \%)$ & $45(92 \%)$ & $\mathrm{p}=0.182$ \\
\hline No & $10(12 \%)$ & $6(21 \%)$ & $4(8 \%)$ & \\
\hline \multicolumn{5}{|c|}{ DMR (at any time) } \\
\hline Yes & $50(64 \%)$ & $13(45 \%)$ & $37(76 \%)$ & $\mathrm{p}=\mathbf{0 . 0 0 2}$ \\
\hline No & $28(36 \%)$ & $16(55 \%)$ & $12(24 \%)$ & \\
\hline
\end{tabular}

Abbreviations: CHR, complete hematological response; CCyR, complete cytogenetic response; EMR, early molecular response; MR3, complete hematological response; DMR, deep molecular response

risk scores did not significantly impact on EFS. Similarly, the achievement of CCyR (either by 6 or by 12 months) did not significantly impact on EFS. On the contrary, the probability of proceeding without events was significantly higher for patients in EMR (94.5\% vs $74.5 \%$ at 24 months; $86.2 \%$ vs $67.8 \%$ at 48 months; $\mathrm{p}=0.021$ ) and for those who achieved a DMR during treatment $(91 \%$ vs $84 \%$ at 24 months; $84 \%$ vs $70 \%$ at 48 months; $p=0.020$ ) (Figure 1 ). It is worth noting that the two groups of patients differed for EMR and DMR rates (Table 1) but not in terms of EUTOS and Sokal scores (Table 2). Therefore, it is plausible that the observed differences between groups could be mainly related to the limited number of patients enrolled.

Moreover, because in these cohorts only one event/ each was censored, we analyzed the EFS in the whole series. When EMR and DMR were considered in the multivariate analysis, both lost the respective statistical significance. No differences in EFS according to the time to MR3 or DMR were observed.

\section{Nilotinib toxicity}

Overall, 45 patients $(57.7 \%)$ developed toxicities: in particular, $10(22.2 \%)$ suffered from hematological and 35 (77.8\%) from extra-hematological adverse events (AEs); 5 cases reported both hematological and extra-hematological toxicities. AEs are listed in Table 3.

Among the extra-hematological toxicities, 30\% interested the skin (more frequently rashes and dry skin), and 25\% pancreas and liver (amylase, lipase, bilirubin and transaminases increased values). Other recorded AEs were metabolic (17.5\%) (hyperglycemia, hypercholesterolemia), vascular (8\%) (two cases of peripheral arterial occlusive disease, one of myocardial ischemia, two case of hypertension, one of arrhythmia), neurological (10\%) (headache), and ocular (5\%) (conjunctivitis) events.

In all cases the hematological toxicities were of grade 1 and 2; the extra-hematological adverse events 
were of grade 1 and 2 in the $48.6 \%$. Only in 2 cases toxicity was of grade 4 (one patient with hepatic toxicity, another one with cutaneous toxicity); in the remaining 16 cases $(45.7 \%)$, the AEs were of grade 3 .

All cases of hematological toxicity occurred in the first year of therapy, with a median time of 1.3 months (range, 1-12); similarly, 80\% of the extra-hematological AEs presented in the first year of treatment, but with a median time of 7.5 months (range, 0.2-85).

It is worth noting that the occurrence of AEs had a significant impact on the drug administration: half of the patients reduced the dose at least once during the follow-

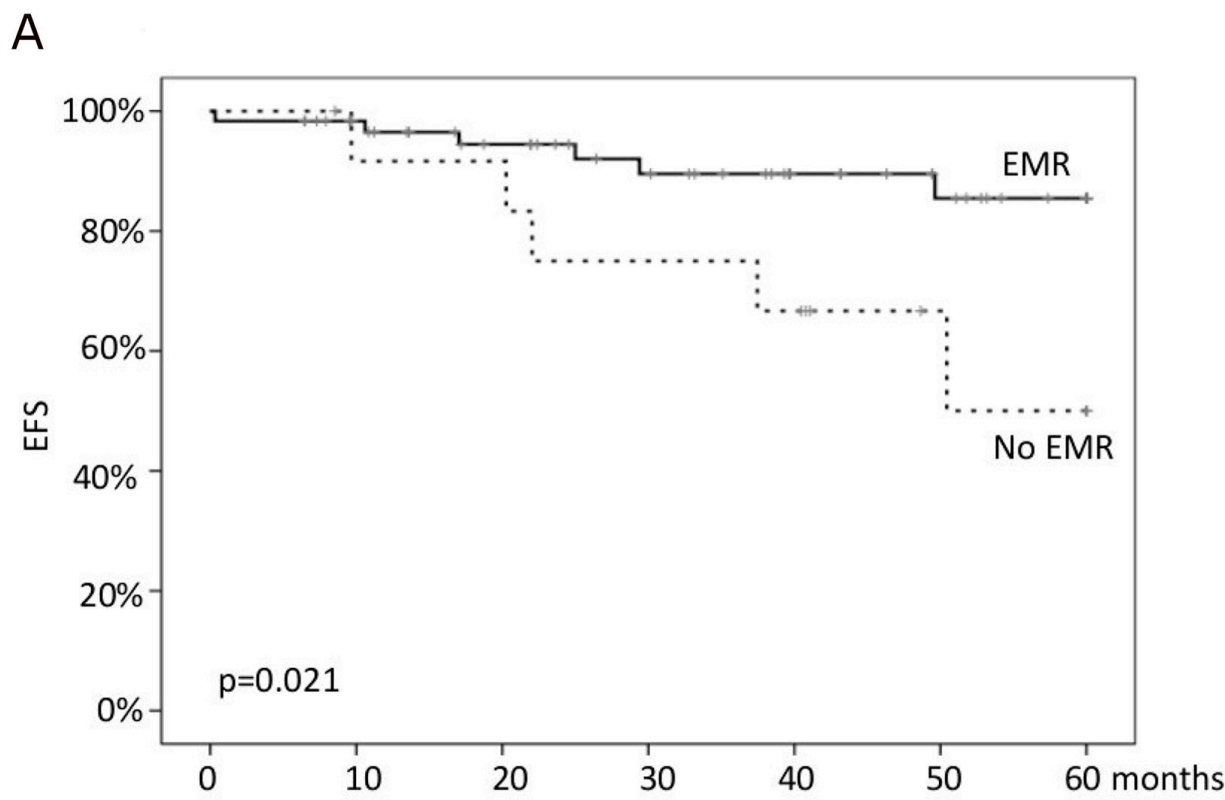

$\begin{array}{lllllll}\text { No. at risk } & & & & & & \\ \text { No EMR } & 13 & 12 & 9 & 9 & 8 & 7 \\ \text { EMR } & 60 & 57 & 55 & 55 & 54 & 54\end{array}$

B

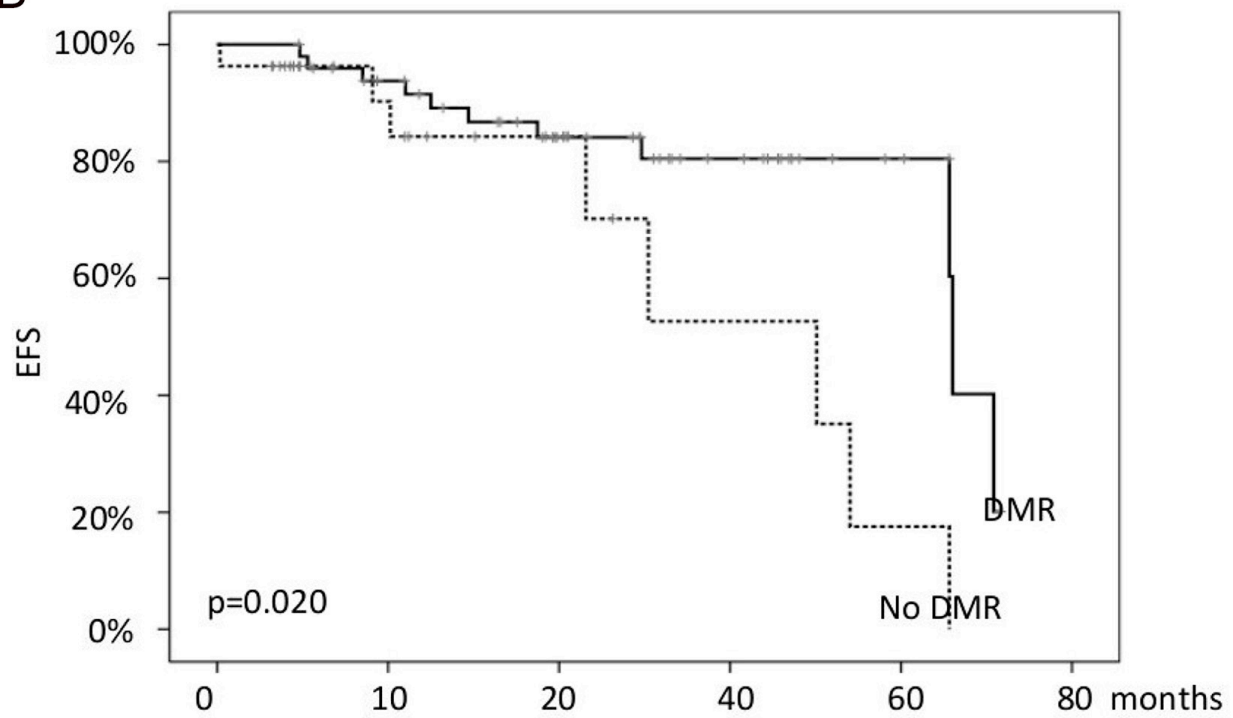

No. at risk

No DMR 26

DMR

49

24

24

22

20

Figure 1: The probability of event-free survival (EFS) of the whole series according to the achievement of early molecular response (EMR, (A)) and deep molecular response (DMR, (B)). 
Table 3: Adverse reactions associated with nilotinib administration (graded according to CTC-AE grading system) and time intervals to events

\begin{tabular}{|c|c|}
\hline TOXICITY & n (\%) \\
\hline \multicolumn{2}{|l|}{ Every toxicity } \\
\hline Yes & $45(58 \%)$ \\
\hline No & $33(42 \%)$ \\
\hline Hematological toxicity & $10(13 \%)$ \\
\hline Grade $1-2$ & $10(100 \%)$ \\
\hline Grade 3 & 0 \\
\hline Grade 4 & 0 \\
\hline \multicolumn{2}{|l|}{ Type of hematological toxicity } \\
\hline Trombocytopenia & 2 \\
\hline Neutropenia & 2 \\
\hline Anemia & 6 \\
\hline Time to hematological toxicity (months)* & $1.3(1-12)$ \\
\hline Extra-hematological toxicity & $35(45 \%)$ \\
\hline Grade $1-2$ & $17(48 \%)$ \\
\hline Grade 3 & $16(46 \%)$ \\
\hline Grade 4 & $2(6 \%)$ \\
\hline \multicolumn{2}{|l|}{ Type of extra-hematological toxicity } \\
\hline Skin toxicity (rash, dry skin, erythema - grade 3,3 pts) & 8 \\
\hline Increase in transaminases (grade 3 in 5 pts), bilirubin (grade 3 in 2 pt) & 8 \\
\hline Increase in amylase/lipase (grade 3 in 4 pts) & 8 \\
\hline Increase in serum glucose/cholesterol & 3 \\
\hline Ocular toxicity (hemorrhage, dry eye, conjunctivitis - grade 3 in 2 pts) & 3 \\
\hline Cardio-vascular toxicity (PAOD - grade 4, hypertension) & 3 \\
\hline CNS toxicity (migrain, stroke - grade 4 ) & 2 \\
\hline Time to extra-hematological toxicity (months) ${ }^{*}$ & $7.5(0.2-85)$ \\
\hline \multicolumn{2}{|l|}{ Dose reduction } \\
\hline Yes & $39(50 \%)$ \\
\hline No & $39(50 \%)$ \\
\hline Time to dose reduction (months)* & $12(0.2-63)$ \\
\hline \multicolumn{2}{|l|}{ Nilotinib definitive discontinuation } \\
\hline Yes & $18(23 \%)$ \\
\hline No & $60(77 \%)$ \\
\hline
\end{tabular}

Notes: *, values are expressed as median (min-max range)

up, and the median time to dose reduction was 12 months (range, 0.2-63).

Finally, 18 patients $(23 \%)$ permanently discontinued treatment: of these, 10 for toxicity, 5 for treatment failure, 2 because of their accrual into suspension trials, and one for personal decision. The median time to discontinuation was 43.5 months (range, 10.6 - 90.8). 
Table 4: Distribution of allele frequencies for investigated polymorphic loci in $A B C B 1$ and $h O C T 1$ genes. For c.2677 locus the $\mathbf{T} / \mathrm{A}$ alleles are indicated as $\mathrm{W}$

\begin{tabular}{|c|c|c|c|c|c|}
\hline Gene and SNP & Genotype & Frequency $\%$ & Allele & Frequency \% & HWE \\
\hline \multirow[t]{3}{*}{$\mathrm{ABCB} 1 \mathrm{c} .1236 \mathrm{C}>\mathrm{T}$} & $\mathrm{CC}$ & 36.0 & \multirow{3}{*}{$\begin{array}{l}\mathrm{C} \\
\mathrm{T}\end{array}$} & \multirow{3}{*}{$\begin{array}{l}59.3 \\
40.7\end{array}$} & \multirow{3}{*}{$\begin{array}{l}\chi^{2}: 0.082 \\
p=0.775\end{array}$} \\
\hline & $\mathrm{CT}$ & 46.7 & & & \\
\hline & $\mathrm{TT}$ & 17.3 & & & \\
\hline \multirow[t]{3}{*}{ ABCB1 c.2677G $>W$} & GG & 40.5 & \multirow{3}{*}{$\begin{array}{l}\mathrm{G} \\
\mathrm{T}\end{array}$} & \multirow{3}{*}{$\begin{array}{l}64.3 \\
35.7\end{array}$} & \multirow{3}{*}{$\begin{array}{l}\chi^{2}: 0.058 \\
p=0.810\end{array}$} \\
\hline & GW & 47.6 & & & \\
\hline & WW & 11.9 & & & \\
\hline \multirow[t]{3}{*}{$\mathrm{ABCB} 1 \mathrm{c.} 3435 \mathrm{C}>\mathrm{T}$} & $\mathrm{CC}$ & 24.0 & \multirow{3}{*}{$\begin{array}{l}\mathrm{C} \\
\mathrm{T}\end{array}$} & \multirow{3}{*}{$\begin{array}{l}50.0 \\
50.0\end{array}$} & \multirow{3}{*}{$\begin{array}{l}\chi^{2}: 0.120 \\
p=0.729\end{array}$} \\
\hline & $\mathrm{CT}$ & 52.0 & & & \\
\hline & $\mathrm{TT}$ & 24.0 & & & \\
\hline \multirow[t]{3}{*}{ hOCT1 c. $480 C>G$} & $\mathrm{CC}$ & 64.0 & \multirow{3}{*}{$\begin{array}{l}\mathrm{C} \\
\mathrm{G}\end{array}$} & \multirow{3}{*}{$\begin{array}{l}79.3 \\
20.7\end{array}$} & \multirow{3}{*}{$\begin{array}{l}\chi^{2}: 0.315 \\
p=0.575\end{array}$} \\
\hline & $\mathrm{CG}$ & 30.7 & & & \\
\hline & GG & 5.3 & & & \\
\hline
\end{tabular}

\section{Transporters' polymorphisms and their clinical significance}

Because genetic polymorphisms of $h O C T 1$ and $A B C B 1$ are associated with variable influx/efflux activity of transporters, the following polymorphisms were evaluated by quantitative PCR: c. $480 \mathrm{C}>\mathrm{G}[\mathrm{hOCTl}]$; c. $1236 \mathrm{C}>\mathrm{T}$, c. $2677 \mathrm{G}>\mathrm{T}$ and c. $3435 \mathrm{C}>\mathrm{T}[A B C B 1]$. In particular, minor allele frequencies (MAFs) for the investigated polymorphisms were comprised in the range 0.207-0.500, and the Hardy-Weinberg equilibrium (HWE) was demonstrated for all single nucleotide polymorphisms (SNPs; Table 4).

The $A B C B 1$ loci were in linkage disequilibrium (range $\mathrm{D}^{\prime}=0.486-0.944$ ), and frequencies of the corresponding haplotypes are reported in Table 5. Interestingly, only $14.2 \%$ of patients were homozygous for the wild-type haplotype (haplotype 1), while $33.3 \%$ were homozygous for the polymorphic haplotypes (haplotypes 2-7).

The $A B C B 1$ [c.1236C $>\mathrm{T}$ ] did not influence the probability of achieving CCyR, EMR, MR3, or DMR or the time to CCyR, MR3, or DMR. The same lack of significance on magnitude or time to responses was found for $A B C B 1$ [c. $2677 \mathrm{G}>\mathrm{T}]$ polymorphism.

On the contrary, the DMR rate was significantly higher for polymorphic c.3435CT/TT patients than for the wild-type c.3435CC ones ( $77.8 \%$ vs $43.0 \%$; $\mathrm{p}=0.02$ ).

Although single $A B C B 1$ polymorphisms returned different results when compared with clinical endpoints, $A B C B 1$ haplotypes were investigated for possible associations with nilotinib efficacy and/or tolerability. The comparisons were made between wild-type (i.e., $\mathrm{CC} / \mathrm{GG} / \mathrm{CC}$ ) and polymorphic haplotypes (any of the other combinations that included polymorphic alleles). In agreement with the results presented above, $A B C B 1$ haplotypes did not significantly condition either the CCyR and MR rate or the time to achieve MR3 or DMR.

Similar findings were obtained for hOCTl, whose genotype at locus c.480 did not exert any influence on CCyR, EMR, MR3 or DMR achievement, or on the time to achieve MR3 or DMR.

Finally, the $A B C B 1$ polymorphisms did not significantly condition the EFS (Figure 2), the presence of toxicities nor their grade, either when analyzed alone or as haplotypes. The same findings were obtained for hOCT1 genotypes with respect to the $\mathrm{c} .480 \mathrm{C}>\mathrm{G}$ polymorphism.

\section{DISCUSSION}

The present study suggests that $A B C B 1$ and $h O C T 1$ transmembrane transporters have not a significant impact on the efficacy and tolerability of nilotinib when used as a first treatment for CML patients. Indeed, our findings clearly show that polymorphisms associated with an altered activity of these transporters do not predict either the clinical outcome or the tolerability. Consequently, we believe that these results could have a clinical output, supporting the use of nilotinib regardless the genetic status of patients with respect of the two considered transporters.

Although the advent of imatinib has dramatically improved the prognosis of CML, about $30 \%$ of patients suspend the therapy for poor efficacy or adverse events [27]. More recently, second-generation (dasatinib, nilotinib, bosutinib) and third-generation TKIs (ponatinib) partially overcame those issues, making possible the successful treatment of resistant/intolerant patients, too $[28,29]$. The choice of the TKI (imatinib, dasatinib or 
Table 5: Distribution of $A B C B 1$ haplotypes (c.1236-c.2677-c.3435) inferred from genotypes of the enrolled patients. For c.2677 locus the T/A alleles are indicated as W

\begin{tabular}{lcc}
\hline [Hapl. ID] & Haplotype definition & Observed frequency \\
\hline $\mathbf{1}$ & CGC & 0.383 \\
{$[2]$} & CGT & 0.224 \\
{$[3]$} & TWT & 0.286 \\
{$[4]$} & TGT & 0.014 \\
{$[5]$} & TGC & 0.023 \\
{$[6]$} & TWC & 0.059 \\
{$[7]$} & CWC & 0.012 \\
\hline
\end{tabular}

nilotinib) as a first-line therapy is sometimes difficult. Generally, the decision is led by the risk score assessment (second-generation TKIs for higher risk scores), age (imatinib for older people), co-morbidities (imatinib or dasatinib for patients with previous cardio-vascular events, nilotinib for those with lung diseases or pulmonary hypertension), but no clear suggestions came from the

A

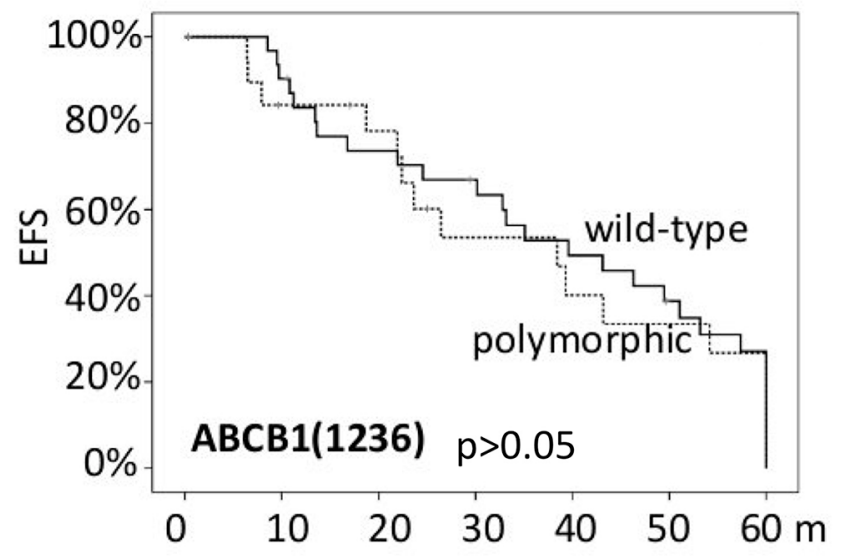

available international guidelines. Therefore, a careful choice of the best TKI $a b$ initio is crucial to reach optimal responses and to reduce the occurrence of adverse events, hence promoting patient's adherence to treatment.

Several attempts to personalize treatment have been made evaluating the predictive role of the polymorphisms in genes coding for transmembrane

$\mathrm{B}$

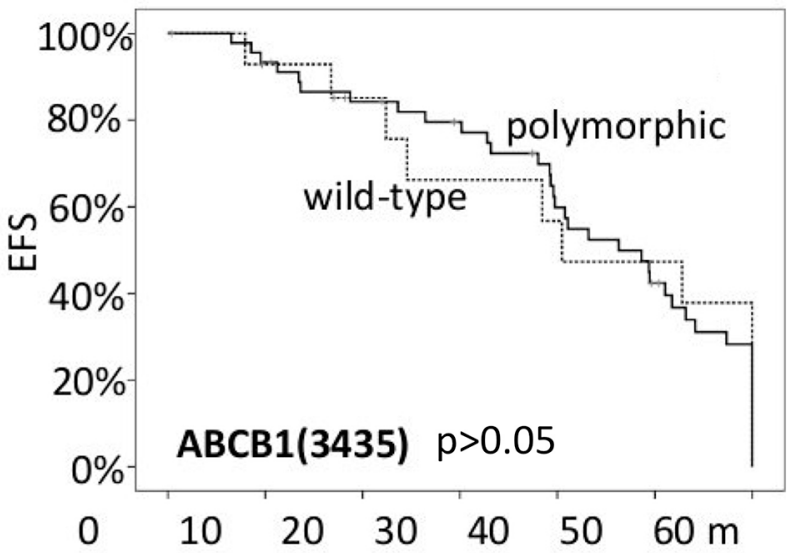

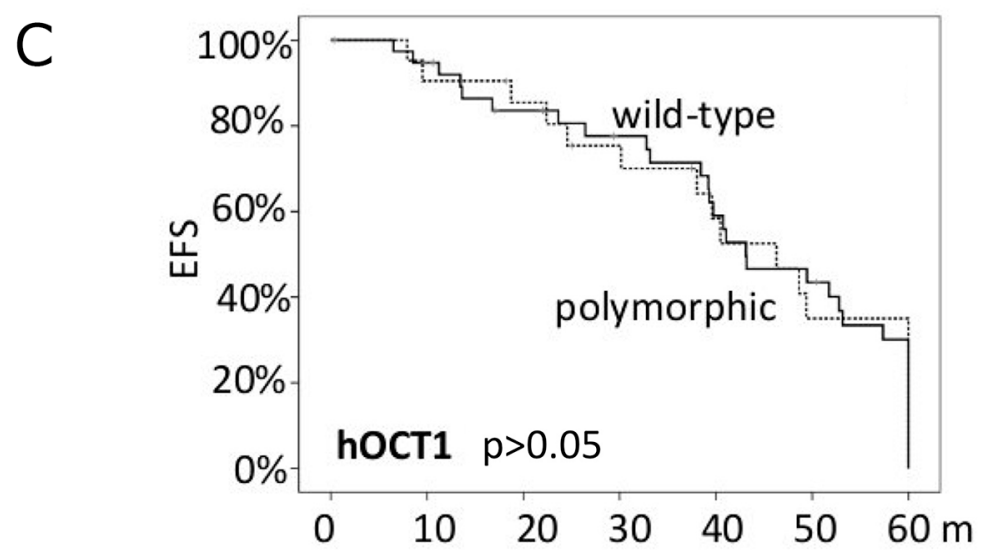

Figure 2: The probability of event-free survival (EFS) according to the ABCB1 polymorphisms (panels $\mathbf{A}$ and $\mathbf{B}$ ) and the hOCT1 polymorphism (panel $\mathbf{C})$. 
transporters. If the picture is clear for imatinib, which is a substrate of both influx (hOCT1) and efflux (ABCB1) transporters, many doubts and contrasting findings characterize second- and third-generation TKIs. For example, the efflux of nilotinib, dasatinib and ponatinib was not significantly influenced by the expression of wild-type or polymorphic $A B C B 1$ in HEK293 and K562 cell lines, differently from imatinib [24]. On the contrary, other authors demonstrated that imatinib, dasatinib, nilotinib were all substrates of ABCB1 in the same K562 cells [25]. Because $A B C B 1$ over-expression significantly reduced when cells were continuously exposed to nilotinib, the authors hypothesized that the entity of the transporter gene expression would be important in the early stages of resistance to TKIs only [30]. Moving from the in vitro models to the clinical setting, other authors showed that the $A B C B 1$ wild-type haplotypes (at positions c.1236, c.2677 and c.3435) were associated with a significantly lower MR3 rate (50\% vs 93\%) under treatment with TKIs. In that small series, only 6 patients received nilotinib, 2 subjects carrying the wild-type and 4 the heterozygous polymorphic haplotype. In contrast with patients receiving imatinib, all cases treated with nilotinib achieved the MR3, regardless their haplotype [31]. The samples size of that study with respect to nilotinib was very limited, but our study showed the same results in a larger group of patients: the wild-type or polymorphic haplotypes did not impact either on the quality of response (CCyR, EMR, MR3, DMR) or on the EFS.

In agreement with what observed for $A B C B 1$, we did not find any clinical impact for the $h O C T 1$ polymorphism on nilotinib efficacy and tolerability; this result was already expected, because it is well known that nilotinib is more lipophilic than imatinib and in vitro experiments suggested that it is not substrate for the hOCT1 influx transporter [32]. Other studies investigated the substrate affinity of nilotinib for other transmembrane transporters, but conclusive results are not yet achieved [33]. The lack of any significant effect of hOCT1 and $A B C B 1$ polymorphisms on the achievement of cytogenetic and molecular responses or on the EFS could be particularly relevant in the clinical practice. Indeed, if a patient carries the hOCT1 polymorphic allele, for example, we can suppose that he will have a higher probability of failure and higher toxicity when exposed to imatinib [17]. In this case, a second-generation TKI, such as nilotinib, might represent a valid option for optimizing the efficacy and reducing the occurrence of AEs.

On the other hand, when patients receive imatinib, it has been reported a higher molecular response rate in the cohort of cases with polymorphic $A B C B 1$ [34]; in our series, $A B C B 1$ polymorphisms did not condition the time to response, its magnitude or the EFS, thus supporting the use of nilotinib independently from the genetic variants of the membrane transporters.
Moreover, our series of patients is really representative of the real clinical practice as witnessed by the superimposable rates of cytogenetic and molecular responses between our study and the ENESTnd trial. Indeed, in that international trial, the CCyR rate was $80 \%$, and $77.2 \%$ of patients achieved an MR3 [4]. In line with those results, the CCyR rate was $88 \%$ in our study, and $77 \%$ of patients achieved an MR3. Furthermore, the 5 -year EFS in our series was lower than that observed in the ENESTnd trial ( $81 \%$ vs $95 \%$, respectively). That difference could depend on the wider definition of EFS in our study, because we considered the loss of MR3 and nilotinib discontinuation for any cause. Indeed, when the same ENESTnd criteria were applied to our series, the 5-year EFS would increase to $94.5 \%$, the same EFS observed in the ENESTnd trial.

Finally, the overall AEs in our study were less frequent than in the ENESTnd trial (41\% vs 60\%); we can suppose that this discrepancy could depend on the prolonged experience in the drug management in our series in respect of the pivotal trial. Nevertheless, the occurrence of the cardiovascular events was similar $(8 \%$ in the ENESTnd vs $7.5 \%$ in our series). Interestingly, in another Italian multicenter "real-life" study on 110 patients, $27 \%$ of them experienced cardiovascular events when nilotinib was administered as second-line treatment [35]. That comparison further strengthens the better tolerability of nilotinib as upfront therapy, despite our study recorded a slight higher discontinuation rate with respect to that reported in the ENESTnd trial (3.8\% vs $2.5 \%$, respectively).

It is worth to note that the demonstrated lack of any significant association between gene polymorphisms of transmembrane transporters and clinical outcome has been found in a homogenous cohort of patients: the fact that all cases received nilotinib as first-line treatment eliminates the possible "contaminating" effects induced by previously used TKIs. Nevertheless, some characteristics of our study have to be considered: first of all, clinical records were available for all patients and they gave the possibility to exclude the risk of drug-drug interactions, as well as smoke, alcohol consumption and comorbidities. On the contrary, the influence of dietary and herbal factors could not be ruled out in a complete manner. Moreover, other patients' characteristics, such as physiological (body mass index, renal and liver function), pathological (comorbidities), genetic (gene polymorphisms, mutations) and epigenetic factors (changes in gene expression) may significantly have an influence on the clinical outcome during nilotinib administration [36]. Although some of those factors have been ruled out thanks to our clinical records, other ones remain unexplored and their future evaluation will require a wider enrollment, further efforts, and probably different analytical platforms, as well as genome sequencing (i.e., next generation sequencing or whole genome association studies). Moreover, our 
database included patients enrolled in retrospective and prospective cohorts, but, as stated above, the strict adoption of the 2013 ELN guidelines for all patients minimized the risk of potential biases. Finally, the present clinical findings are similar or superimposable with those reported in the ENESTnd trial as stated above, thus strengthening the reliability of our spontaneous and cooperative study.

In conclusion, for the first time our study supports the use of nilotinib as first-line treatment in a homogeneous series of CML patients irrespective to the hOCT1 and $A B C B 1$ genotypes, replicating data obtained in the largest, company-driven ENESTnd trial. In contrast to these findings, our group recently published the significant influence of ABCB1/hOCT1 combined genotype on imatinib efficacy and tolerability [37]. Therefore, a simple and cheap test, such as a quantitative PCR, would aid the physician to correctly choose the right TKI for each patient, even if the test could not be available for all patients. Indeed, when a reduced cellular uptake through hOCT1 and higher efflux by ABCB1 do increase the risk of imatinib failure, nilotinib may represent the optimal choice.

\section{MATERIALS AND METHODS}

The current study is part of a spontaneous, not sponsored sub-study of the "TIKLET" trial (Tyrosine Kinase Inhibitors in chronic myeloid leukemia: Efficacy and Tolerability; ClinicalTrials.gov identifier: NCT01860456), which has been carried out on 78 adult subjects affected by CML in chronic phase receiving nilotinib $600 \mathrm{mg}$ as first-line treatment, outside clinical trials, in 8 Italian Hematology Divisions. Of note, none of the enrolled patients took part in previous clinical trials.

From January 2013 to July 2015, 29 patients were prospectively enrolled in the study. Sample size analysis demonstrated the study was downsized, hence further 49 subjects receiving first-line nilotinib after 2008 were also considered if a) they received nilotinib as first-line treatment and b) the standardized $B C R-A B L 1 / A B L 1$ quantitative PCR was available, according to the Labnet Italian Network standardized operative procedures. Those criteria allowed the complete revision of patients' clinical history and laboratory results (see following paragraphs), hence statistical analyses were performed in a homogeneous population with respect to clinical and molecular endpoints. Statistical analyses between groups were performed only to exclude severe differences.

The study protocol has been approved by the Ethics Committee of the Azienda Ospedaliero Universitaria Pisana (protocol n. 46013/2011) and by local Ethics Committees of participating centres. Patients gave their informed consent to study participation before their enrollment.
The following inclusion criteria were adopted: (a) patients of both gender, (b) aged $\geq 18$ years, (c) treated with nilotinib as first-line treatment, (d) with at least 3 months of follow-up, (e) the availability of standardized $B C R-A B L 1 / A B L 1$ quantitative PCR results both at diagnosis and during the follow-up; (f) complete clinical history of patients.

All patients were treated with an initial daily dose of $600 \mathrm{mg}$.

Age, sex, Sokal, Hasford and EUTOS risk scores were recorded; for each patient, two independent hematologists scored the hematological, cytogenetic and molecular responses defined according to the ELN recommendations edited in 2013 [26]. In this manner, every bias in scoring the clinical efficacy of nilotinib was kept at a minimum (see below).

The AEs data collection has been done according to the Common Toxicity Criteria -Adverse Events (CTC$\mathrm{AE})$ version 4.03. In agreement with the efficacy data, the database of toxicities was deeply reviewed by two hematologists according to the above-cited CTC-AE criteria.

\section{$B C R-A B L 1 / A B L 1$ response monitoring}

Hematological, cytogenetic, and molecular responses were assessed and classified according to the ELN guidelines [26]. Furthermore, molecular monitoring was carried out on peripheral blood samples and performed by quantitative PCR, as established by the European guidelines [38] applied by all Italian laboratories afferent to the LABNET network, and results expressed in accordance with the International Scale (IS). In particular, molecular responses were "graded" according to the logarithmic reduction with respect to a basal level (considered as 100\%). Thus, the molecular responses MR3, MR4 and MR5 imply 3-log (0.1\% IS), 4-log (0.01\% IS) and $5-\log (0.001 \%$ IS) reduction, respectively. The responses with a $\geq 4-\log$ decrease identified the DMR, the sensitivity of assays depending on the number of the $A B L 1$ copies in each amplification well (always $>10.000$ ). Finally, the EMR was defined as a $B C R-A B L 1 / A B L 1 \%$ IS $\leq 10 \%$ at 3 months from the treatment beginning.

\section{Pharmacogenetic analysis}

The genetic variables considered were represented by the following polymorphisms: a) hOCT1 rs683369 [c.480C $>\mathrm{G}$ ]; b) $A B C B 1$ rs 1128503 [c.1236C $>\mathrm{T}$ ] (exon 12); c) $A B C B 1$ rs2032582 [c.2677G $>\mathrm{T} / \mathrm{A}]$ (exon 21); d) $A B C B 1$ rs 1045642 [c.3435C $>$ T] (exon 26).

Genomic DNA extracted from peripheral blood (QIAamp DNA Blood Mini Kit, Qiagen, Milan, Italy) was used to obtain the genotype of each patient by using specific TaqMan assays (Life Sciences, Milan, Italy) on 
an ABI Prism 7900HT Sequence Detection System (Life Sciences).

MAF calculation, HWE evaluation and haplotype analyses were performed by using Arlequin software [39]. The recessive model was adopted, grouping patients according to the absence (wild-type) or the presence (heterozygous or polymorphic homozygous) of at least one polymorphic allele for each locus.

\section{Statistical analysis}

The diagnoses and the corresponding followup periods for the retrospective cohort were reviewed independently by two hematologists, according to the new ELN recommendations [26]. That approach gave the possibility to gather together clinical records from both retrospective and prospective cohort of patients, without increasing the risk of potential biases.

For the main clinical variables, central indices (mean, median) and dispersion parameters $(\mathrm{SD}$, range) were obtained. The analysis of variance (ANOVA) was used to evaluate every possible association between clinical efficacy measures and genetic variables. The Fisher's exact test was applied for the analysis of discrete variables, while the $t$-test was used for comparing the time to cytogenetic or molecular response and dichotomous variables.

The EFS was assessed from the date of initiation of treatment to the onset of any of the following events: absence of CCyR at 12 months, loss of CCyR and MR3 at any time during treatment, withdrawal of the drug for toxicity, progression to the accelerated/blast phase, any cause of death during the study. The probability of EFS was calculated using the Kaplan-Meier method.

Overall, the level of significance was set at $\mathrm{p}<0.05$.

\section{Abbreviations}

ABC, ATP binding cassette; AE: adverse event; CCyR: complete cytogenetic response; CHR: complete hematological response; CML: chronic myeloid leukemia; CTC-AE, Common Toxicity Criteria-Adverse Events; DMR: deep molecular response; ELN, European Leukemia Net; EFS: event-free survival; EMR: early molecular response; EUTOS, European Treatment and Outcome Study; hOCT1, human organic cation transport member 1; HWE: Hardy-Weinberg equilibrium; IS: international scale; MAF: minor allele frequency; MR3: major molecular response; SNP: single nucleotide polymorphism; TKI: tyrosine kinase inhibitor.

\section{Author contributions}

SG and ADP conceived and planned the study and, in cooperation with MP and RD, supervised the study. SG, CB, CF, FG, GRC, AI, FL, GC, MG, MB, LA, AG, BS, DC, ARS, GLN, AB, PL, GS, GV, AC, MP,
AI were involved in patients' enrollment and clinical data collection. EA, SG, FG, EC and AD'A performed molecular assessments. All authors wrote, revised and approved the final version of the manuscript.

\section{ACKNOWLEDGMENTS}

We are grateful to the patients who participated in this study and the nurse staff for their precious help. We would like to thank the Associazione Italiana per lo studio delle Leucemie e Linfomi (AIL) and DG, GM, ML, MM, $\mathrm{AB}, \mathrm{CG}$, and $\mathrm{EB}$ for their support to our researches.

\section{CONFLICTS OF INTEREST}

SG, ADP, AI, GS, GRC, MP, CF participated to advisory boards sponsored by Novartis. Nevertheless, all authors declare no conflicts of interest concern this spontaneous, not industry-sponsored study.

\section{FUNDING}

This work was supported by funds from the authors' institutions, and in particular by funds from the University of Pisa (PRA 2015 grant) to ADP.

\section{REFERENCES}

1. Castagnetti F, Di Raimondo F, De Vivo A, Spitaleri A, Gugliotta G, Fabbiano F, Capodanno I, Mannina D, Salvucci M, Antolino A, Marasca R, Musso M, Crugnola $\mathrm{M}$, et al. A population-based study of chronic myeloid leukemia patients treated with imatinib in first line. Am J Hematol. 2017; 92: 82-7.

2. Kantarjian HM, Hochhaus A, Saglio G, De Souza C, Flinn IW, Stenke L, Goh YT, Rosti G, Nakamae H, Gallagher NJ, Hoenekopp A, Blakesley RE, Larson RA, et al. Nilotinib versus imatinib for the treatment of patients with newly diagnosed chronic phase, Philadelphia chromosomepositive, chronic myeloid leukaemia: 24-month minimum follow-up of the phase 3 randomised ENESTnd trial. Lancet Oncol. 2011; 12: 841-51.

3. O'Hare T, Walters DK, Deininger MW, Druker BJ. AMN107: tightening the grip of imatinib. Cancer Cell. 2005; 7 : 117-9.

4. Hochhaus A, Saglio G, Hughes TP, Larson RA, Kim DW, Issaragrisil S, le Coutre PD, Etienne G, Dorlhiac-Llacer PE, Clark RE, Flinn IW, Nakamae H, Donohue B, et al. Longterm benefits and risks of frontline nilotinib vs imatinib for chronic myeloid leukemia in chronic phase: 5-year update of the randomized ENESTnd trial. Leukemia. 2016; 30: 1044-54.

5. Soverini S, De Benedittis C, Polakova KM, Linhartova J, Castagnetti F, Gugliotta G, Papayannidis C, Mancini M, Klamova H, Salvucci M, Crugnola M, Iurlo A, Albano F, 
et al. Next-generation sequencing for sensitive detection of BCR-ABL1 mutations relevant to tyrosine kinase inhibitor choice in imatinib-resistant patients. Oncotarget. 2016; 7: 21982-90. https://doi.org/10.18632/oncotarget.8010.

6. Riva B, De Dominici M, Gnemmi I, Mariani SA, Minassi A, Minieri V, Salomoni P, Canonico PL, Genazzani AA, Calabretta B, Condorelli F. Celecoxib inhibits proliferation and survival of chronic myelogeous leukemia (CML) cells via AMPK-dependent regulation of $\beta$-catenin and mTORC1/2. Oncotarget. 2016; 7: 81555-70. https://doi. org/10.18632/oncotarget.13146.

7. Wang LX, Wang JD, Chen JJ, Long B, Liu LL, Tu XX, Luo Y, Hu Y, Lin DJ, Lu G, Long ZJ, Liu Q. Aurora A Kinase Inhibitor AKI603 Induces Cellular Senescence in Chronic Myeloid Leukemia Cells Harboring T315I Mutation. Sci Rep. 2016; 6: 35533.

8. Eiring AM, Page BD, Kraft IL, Mason CC, Vellore NA, Resetca D, Zabriskie MS, Zhang TY, Khorashad JS, Engar AJ, Reynolds KR, Anderson DJ, Senina A, et al. Combined STAT3 and BCR-ABL1 inhibition induces synthetic lethality in therapy-resistant chronic myeloid leukemia. Leukemia. 2015; 29: 586-97.

9. Christiansson L, Söderlund S, Svensson E, Mustjoki S, Bengtsson M, Simonsson B, Olsson-Strömberg U, Loskog AS. Increased level of myeloid-derived suppressor cells, programmed death receptor ligand 1/programmed death receptor 1, and soluble CD25 in Sokal high risk chronic myeloid leukemia. PLoS One. 2013; 8: e55818.

10. Arrigoni E, Galimberti S, Petrini M, Danesi R, Di Paolo A. ATP-binding cassette transmembrane transporters and their epigenetic control in cancer: an overview. Expert Opin Drug Metab Toxicol. 2016; 12: 1419-32.

11. Declèves X, Bihorel S, Debray M, Yousif S, Camenisch G, Scherrmann JM. ABC transporters and the accumulation of imatinib and its active metabolite CGP74588 in rat C6 glioma cells. Pharmacol Res. 2008; 57: 214-22.

12. Staud F, Ceckova M, Micuda S, Pavek P. Expression and function of p-glycoprotein in normal tissues: effect on pharmacokinetics. Methods Mol Biol. 2010; 596: 199-222.

13. Jiang ZP, Zhao XL, Takahashi N, Angelini S, Dubashi $\mathrm{B}$, Sun $\mathrm{L}, \mathrm{Xu} \mathrm{P}$. Trough concentration and ABCG2 polymorphism are better to predict imatinib response in chronic myeloid leukemia: a meta-analysis. Cancer Lett. 2013; 328: 307-17.

14. Vaidya S, Ghosh K, Shanmukhaiah C, Vundinti BR. Genetic variations of hOCT1 gene and CYP3A4/A5 genes and their association with imatinib response in Chronic Myeloid Leukemia. Eur J Pharmacol. 2015; 765: 124-30.

15. Vine J, Cohen SB, Ruchlemer R, Goldschmidt N, Levin M, Libster D, Gural A, Gatt ME, Lavie D, Ben-Yehuda D, Rund D. Polymorphisms in the human organic cation transporter and the multidrug resistance gene: correlation with imatinib levels and clinical course in patients with chronic myeloid leukemia. Leuk Lymphoma. 2014; 55: 2525-31.

16. Di Paolo A, Polillo M, Capecchi M, Cervetti G, Baratè C, Angelini S, Guerrini F, Fontanelli G, Arici R, Ciabatti E, Grassi S, Bocci G, Hrelia P, et al. The c.480C $>$ G polymorphism of hOCT1 influences imatinib clearance in patients affected by chronic myeloid leukemia. Pharmacogenomics J. 2014; 14: 328-35.

17. Kim YK, Lee SS, Jeong SH, Ahn JS, Yang DH, Lee JJ, Shin MG, Kim HJ. OCT-1, ABCB1, and ABCG2 Expression in Imatinib-Resistant Chronic Myeloid Leukemia Treated with Dasatinib or Nilotinib. Chonnam Med J. 2014; 50: 102-11.

18. Kosztyu P, Bukvova R, Dolezel P, Mlejnek P. Resistance to daunorubicin, imatinib, or nilotinib depends on expression levels of $\mathrm{ABCB} 1$ and $\mathrm{ABCG} 2$ in human leukemia cells. Chem Biol Interact. 2014; 219: 203-10.

19. Hiwase DK, Saunders V, Hewett D, Frede A, Zrim S, Dang P, Eadie L, To LB, Melo J, Kumar S, Hughes TP, White DL. Dasatinib cellular uptake and efflux in chronic myeloid leukemia cells: therapeutic implications. Clin Cancer Res. 2008; 14: 3881-8.

20. Hegedus C, Ozvegy-Laczka C, Apáti A, Magócsi M, Német K, Orfi L, Kéri G, Katona M, Takáts Z, Váradi A, Szakács G, Sarkadi B. Interaction of nilotinib, dasatinib and bosutinib with $\mathrm{ABCB} 1$ and $\mathrm{ABCG}$ : implications for altered anti-cancer effects and pharmacological properties. Br J Pharmacol. 2009; 158: 1153-64.

21. Dessilly G, Panin N, Elens L, Haufroid V, Demoulin JB. Impact of $\mathrm{ABCB} 11236 \mathrm{C}>\mathrm{gt}$; $\mathrm{T}-2677 \mathrm{G}>\mathrm{gt}$; T-3435C $>\mathrm{gt}$; T polymorphisms on the anti-proliferative activity of imatinib, nilotinib, dasatinib and ponatinib. Sci Rep. 2016; 6: 29559.

22. Redaelli S, Perini P, Ceccon M, Piazza R, Rigolio R, Mauri M, Boschelli F, Giannoudis A, Gambacorti-Passerini C. in vitro and in vivo identification of $\mathrm{ABCB} 1$ as an efflux transporter of bosutinib. J Hematol Oncol. 2015; 8: 81.

23. Tiwari AK, Sodani K, Dai CL, Abuznait AH, Singh S, Xiao ZJ, Patel A, Talele TT, Fu L, Kaddoumi A, Gallo JM, Chen ZS. Nilotinib potentiates anticancer drug sensitivity in murine ABCB1-, ABCG2-, and ABCC10-multidrug resistance xenograft models. Cancer Lett. 2013; 328: 307-17.

24. Dulucq S, Bouchet S, Turcq B, Lippert E, Etienne G, Reiffers J, Molimard M, Krajinovic M, Mahon FX. Multidrug resistance gene (MDR1) polymorphisms are associated with major molecular responses to standard-dose imatinib in chronic myeloid leukemia. Blood. 2008; 112: 2024-7.

25. Angelini S, Soverini S, Ravegnini G, Barnett M, Turrini E, Thornquist M, Pane F, Hughes TP, White DL, Radich J, Kim DW, Saglio G, Cilloni D, et al. Association between imatinib transporters and metabolizing enzymes genotype and response in newly diagnosed chronic myeloid leukemia patients receiving imatinib therapy. Haematologica. 2013; 98: 193-200. 
26. Baccarani M, Deininger MW, Rosti G, Hochhaus A, Soverini S, Apperley JF, Cervantes F, Clark RE, Cortes JE, Guilhot F, Hjorth-Hansen H, Hughes TP, Kantarjian HM, et al. European LeukemiaNet recommendations for the management of chronic myeloid leukemia: 2013. Blood. 2013; 122: 872-84.

27. Hoffmann VS, Baccarani M, Hasford J, Castagnetti F, Di Raimondo F, Casado LF, Turkina A, Zackova D, Ossenkoppele G, Zaritskey A, Höglund M, Simonsson B, Indrak K, et al. Treatment and outcome of 2904 CML patients from the EUTOS population-based registry. Leukemia. 2017; 31: 593-601.

28. Cortes JE, Khoury HJ, Kantarjian HM, Lipton JH, Kim DW, Schafhausen P, Matczak E, Leip E, Noonan K, Brümmendorf TH, Gambacorti-Passerini C. Long-term bosutinib for chronic phase chronic myeloid leukemia after failure of imatinib plus dasatinib and/or nilotinib. Am J Hematol. 2016; 91: 1206-14.

29. Poch Martell M, Sibai H, Deotare U, Lipton JH. Ponatinib in the therapy of chronic myeloid leukemia. Expert Rev Hematol. 2016; 9: 923-32.

30. Eadie LN, Hughes TP, White DL. ABCB1 Overexpression Is a Key Initiator of Resistance to Tyrosine Kinase Inhibitors in CML Cell Lines. PLoS One. 2016; 11: e0161470.

31. Lardo M, Castro M, Moiraghi B, Rojas F, Borda N, Rey JA, Lazarowski A. MDR1/ABCB1 gene polymorphisms in patients with chronic myeloid leukemia. Blood Res. 2015; 50: $154-9$.

32. Blay JY, von Mehren M. Nilotinib: a novel, selective tyrosine kinase inhibitor. Semin Oncol. 2011; 38: S3-9.

33. Polillo M, Galimberti S, Baratè C, Petrini M, Danesi R, Di Paolo A. Pharmacogenetics of BCR/ABL Inhibitors in Chronic Myeloid Leukemia. Int J Mol Sci. 2015; 16: 22811-29.

34. Salimizand H, Amini S, Abdi M, Ghaderi B, Azadi NA. Concurrent effects of ABCB1 C3435T, ABCG2 C421A, and XRCC1 Arg194Trp genetic polymorphisms with risk of cancer, clinical output, and response to treatment with imatinib mesylate in patients with chronic myeloid leukemia. Tumour Biol. 2016; 37: 791-8.

35. Bocchia M, Galimberti S, Aprile L, Sicuranza A, Gozzini A, Santilli F, Abruzzese E, Baratè C, Scappini B, Fontanelli G, Trawinska MM, Defina M, Gozzetti A, et al. Genetic predisposition and induced pro-inflammatory/pro-oxidative status may play a role in increased atherothrombotic events in nilotinib treated chronic myeloid leukemia patients. Oncotarget. 2016; 7: 72311-21. https://doi.org/10.18632/ oncotarget.11100.

36. Crea F, Di Paolo A, Liu HH, Polillo M, Clermont PL, Guerrini F, Ciabatti E, Ricci F, Baratè C, Fontanelli G, Barsotti S, Morganti R, Danesi R, et al. Polycomb genes are associated with response to imatinib in chronic myeloid leukemia. Epigenomics. 2015; 7: 757-65.

37. Galeotti L, Ceccherini F, Domingo D, Laurino M, Polillo M, Di Paolo A, Baratè C, Fava C, D'Avolio A, Cervetti G, Guerrini F, Fontanelli G, Ciabatti E, et al. Association of the hOCT1/ABCB1 genotype with efficacy and tolerability of imatinib in patients affected by chronic myeloid leukemia. Cancer Chemother Pharmacol. 2017; 79: 767-773.

38. Cross NC, White HE, Colomer D, Ehrencrona H, Foroni L, Gottardi E, Lange T, Lion T, Machova Polakova K, Dulucq S, Martinelli G, Oppliger Leibundgut E, Pallisgaard N, et al. Laboratory recommendations for scoring deep molecular responses following treatment for chronic myeloid leukemia. Leukemia. 2015; 29: 999-1003.

39. Excoffier L, Lischer HE. Arlequin suite ver 3.5: a new series of programs to perform population genetics analyses under Linux and Windows. Mol Ecol Resour. 2010; 10: 564-7. 